\title{
Anti Hemolytic Activity of Rutin incase of Phenyl Hydrazine induced Hemolysis
}

\author{
Shree Ramani ${ }^{1}$, Satodeepa Basak ${ }^{1}$, Puneeth $\mathbf{S}^{1}$, Karthick Mahato ${ }^{1}$ and Malathi $\mathbf{R}^{2^{*}}$ \\ ${ }^{1}$ Department of Biochemistry, Indian Academy Degree College Autonomous \\ $2^{\star}$ Corresponding Author, Department of Life Sciences, Kristu Jayanti College Autonomous
}

\begin{abstract}
The important role of Red Blood Cells (RBCs) is to transport oxygen to the tissues from the lungs to provide oxygen to all cells. During circulation, RBCs are continuously exposed to both endogenous and exogenous sources of reactive oxygen species (ROS) that can injury the RBC and ruin its function. The imbalance between cellular production of reactive oxygen species and the counteracting antioxidant mechanism leads to Oxidative stress. Flavonoids are low molecular weight secondary polyphenolic metabolites present in plants characterized by their flavan nucleus. Rutin is a flavonol plentiful in a variety of commonly ingested foods. The term 'Rutin' came from a plant known as Ruta graveolens that also encompasses Rutin. Teas and fruits have high concentration of Rutin. Buckwheat seeds (Fagopyrum esculantum) are the richest source. Rutin protects the body from cellular damage caused by free radicals with its antioxidant properties. It helps to eliminate cholesterol from the body and increase elasticity of the arterial walls, which in turn promotes greater blood flow. The present investigation was aimed to assess the antihemolytic activity of the Rutin using phenyl hydrazine as hemolysin. This study uses RBC as model system to access the anti-hemolytic activity of Rutin using phenyl hydrazine as hemolysin. Phenyl hydrazine which was used previously in treating sickle cell anemia was used in this investigation as hemolysin. The result of the study shows that the addition of phenyl hydrazine $[1-500 \mu \mathrm{g} / \mathrm{ml}]$ to the RBC suspension caused significant $(P<0.01)$ rise in hemolysis. The cell pellet in the bottom of the tubes reduced to reddish colored supernatant indicating hemolysis. The effect was concentration dependent. The present investigation clearly indicates that phenyl hydrazine causes hemolysis and toxicity to RBC. The concurrent addition of phenyl hydrazine along with the test compound Rutin $(100-500 \mu \mathrm{g} / \mathrm{ml})$ to the RBC suspension significantly $(P<0.01)$ reduced phenyl hydrazine induced hemolysis in a concentration dependent manner. The protective effect of Rutin on the hemolysis induced by phenyl hydrazine clearly shows it acts as anti-hemolytic agent. This finding was compared with the standard ascorbic acid. IC50 value of Rutin and ascorbic acid were 160.86 and $163.5 \mu \mathrm{g} / \mathrm{ml}$ respectively. The Rutin extract showed maximum inhibitory effect $69.8 \%$.at $500 \mu \mathrm{g} / \mathrm{ml}$. This shows that Rutin can be effectively used as anti-hemolytic agent.
\end{abstract}

Keyswords Hemolysis, Rutin, Phenyl hydrazine, Antihemolytic agent, ROS, RBC's

\section{Introduction}

Oxidative stress occurs when the formation of free radicals and active intermediates in a biological system surpasses its capability to neutralize and remove them. Reactive oxygen intermediate (ROI) and reactive nitrogen intermediate $(\mathrm{RNI})$ are constantly produced under physiological conditions is the crucial event in living organisms. The concept of oxidative stress limited to ROI such as hydroxyl and superoxide radicals, and hydrogen peroxide and singlet oxygen has been added onto RNI such as nitric oxide (NO), per-oxynitrite and, recently, to $S$-nitrosothiols. Thus, ROI and RNI react with proteins, carbohydrates and lipids, with consequent alteration both in the intracellular and intercellular homeostasis, leading to possible cell death and regeneration [Taibur Rahman et al., 2012]. Oxidative stress is implicated in several mental disorders including depression, anxiety disorders, schizophrenia and bipolar disorder [Samina salim et al, 2014].

To manage with the oxidative stress elicited by aerobic metabolism, animal and human cells have established a ubiquitous antioxidant defense system, which consists of superoxide dismutase (SOD), catalase (CAT), glutathione peroxidase (GPx) and glutathione reductase together with a number of low molecular weight antioxidants such as flavinoids, ascorbate, $\alpha$-tocopherol and glutathione, cysteine, thioredoxin, vitamins, etc. Though, this antioxidant defense system may be overcome by numerous pathological or environmental factors so that a portion of ROS may escape destruction and form the far more reactive hydroxyl radicals. An increase in ROS provoked oxidative damage to DNA and other biomolecules may damage normal functions of tissue cells and lead to human aging and disease. The present investigation was aimed to assess the antihemolytic activity of the Test compound Rutin using RBC as model system.

\section{Materials and Methods:}

\section{Chemicals:}

Sodium chloride, Phenyl Hydrazine, Ascorbic acid , Rutin(From Banashankari scientific, ASVNV Bhavan, Kempegowda road, Bangalore-09) 


\section{INVESTIGATION OF ANTI-HEMOLYTIC ACTIVITY:}

\section{Blood Sample:}

The normal; anti-coagulated blood was collected from the Poly vista, Bangalore.

\section{Preparation of RBC cell suspension:}

The collected anti-coagulated blood was washed several times with phosphate buffered saline to remove (protein) Buffy coat. $3 \mathrm{ml}$ of anti-coagulated blood was mixed with $10 \mathrm{ml}$ of phosphate buffered saline and then centrifuged for 5 minutes at $1500-1800 \mathrm{rpm}$. The supernatant was discarded. $10 \mathrm{ml}$ of phosphate buffered saline was added to the pellet, centrifuged and discarded the supernatant. This washing was repeated for 3-4 times. Total volume of RBC was found by the formula,

Total volume $=$ packed cell volume/designed cell concentration $\times 100$

Using the total volume, RBC suspension preparation was prepared

RBC suspension $=$ Total volume of the RBC suspension volume of packed cell

The suspension was prepared at a concentration of $5 \%$ by using phosphate buffered saline.

\section{Amelioration of phenyl hydrazine induced hemolysis:}

To evaluate the efficacy of rutin in amelioration of phenyl hydrazine induced toxicity on human RBC, 4 sets of tubes containing $0.1 \mathrm{ml}$ of RBC suspension were prepared as mentioned below:

1. Control tubes containing only RBC suspension.

2. Tubes containing RBC suspension and phenyl hydrazine (1 to $700 \mu \mathrm{g}$ ).

3. Control tubes containing RBC suspension and test compound $(100-500 \mu \mathrm{g})$

4. Tubes containing RBC suspension and phenyl hydrazine $(500 \mu \mathrm{g})$ with varying concentration of test compound (100 to $500 \mu \mathrm{g}$ ).

The volume of all tube is made up to $2 \mathrm{ml}$ with phosphate buffered saline in methodical to have the equal volume in all the tubes. All the tubes were mixed gently and incubated at $37^{\circ} \mathrm{C}$ for 4 hours with intermittent shaking. After that the tubes were centrifuged at $1000 \mathrm{~g}$ for 10 minutes and the colour density of the supernatant was measured spectrophotometrically at $540 \mathrm{~nm}$. The percent hemolysis was calculated using the formula below:

Percent hemolysis $=$ Absorbance of the individual tube $\times 100$

$$
\text { Absorbance with } 100 \% \text { hemolysis }
$$

To attain 100 percent hemolysis, $1.9 \mathrm{ml}$ of distilled water was added to $0.1 \mathrm{ml}$ of RBC suspension. The percent retardation of test compound was calculated using the formula:

Percent retardation $=\underline{A-B} \times 100$

A

Where, $\quad A=$ phenyl hydrazine induced hemolysis.

$\mathrm{B}=$ hemolysis caused by concurrent addition of phenyl hydrazine and test compound.

\section{Statistical_Analysis}

Test was carried out in triplicate. All results are expressed as mean \pm S.E.M. Statistical analysis was performed using ANOVA. P-values less than 0.01 were considered statistical significant. Linear regression analysis was used to calculate the $L_{5}$ and $I C_{50}$ values.

\section{Result}

Results shown in table 1 indicate that addition of phenyl hydrazine $[1-500 \mu \mathrm{g} / \mathrm{ml}]$ to the RBC suspension caused significant $(P<0.01)$ rise in hemolysis. The cell pellet in the bottom of the tubes reduced to reddish colored supernatant indicating hemolysis. The effect was concentration dependent. The present investigation clearly indicates that phenyl hydrazine causes hemolysis and toxicity to RBC. [Table 1]

Table-1: Phenyl hydrazine induced hemolysis

\begin{tabular}{|c|c|c|c|}
\hline S.No & $\begin{array}{c}\text { Concentration } \\
(\mu \mathrm{g} / \mathrm{ml})\end{array}$ & OD & $\begin{array}{c}\% \\
\text { hemolysis }\end{array}$ \\
\hline 1 & 1 & $0.03 \pm 0.009$ & $7.41 \pm 3.17$ \\
\hline 2 & 5 & $0.026 \pm 0.012$ & $8.38 \pm 4.39$ \\
\hline 3 & 10 & $0.030 \pm 0.139$ & $9.67 \pm 4.56$ \\
\hline 4 & 100 & $0.033 \pm 0.014$ & $10.64 \pm 3.83$ \\
\hline 5 & 200 & $0.036 \pm 0.014$ & $11.61 \pm 4.65$ \\
\hline 6 & 300 & $0.040 \pm 0.0163$ & $12.90 \pm 5.27$ \\
\hline 7 & 400 & $0.050 \pm 0.0163$ & $16.12 \pm 5.27$ \\
\hline 8 & 500 & $0.110 \pm 0.039$ & $35.48 \pm 12.44$ \\
\hline 9 & 600 & $0.170 \pm 0.034$ & $40.53 \pm 3.25$ \\
\hline 10 & 700 & $0.170 \pm 0.034$ & $40.53 \pm 3.25$ \\
\hline LC $_{50}=832.35 \mu \mathrm{g}$ & & \\
\hline
\end{tabular}

The concurrent addition of phenyl hydrazine along with the test compound Rutin $(100-500 \mu \mathrm{g} / \mathrm{ml})$ to the RBC suspension significantly $(P<0.01)$ reduced phenyl hydrazine induced hemolysis. As shown in table 2 the effect was concentration dependent.

The protective effects of Rutin extract and reference standard ascorbic acid on the hemolysis induced by phenyl hydrazine are shown in figure presenting 
the percentage of hemolysis inhibition at various concentrations. $\mathrm{IC}_{50}$ of the Rutin extract and ascorbic acid were 160.86 and $163.5 \mu \mathrm{g} / \mathrm{ml}$ respectively. The Rutin extract showed maximum inhibitory effect $69.8 \%$. at $500 \mu \mathrm{g} / \mathrm{ml}$.

Table-2: Effect of the test compound Rutin on phenyl hydrazine induced hemolysis.

\begin{tabular}{|c|c|c|c|c|}
\hline S.No & $\begin{array}{c}\text { Concentration } \\
(\mu \mathrm{g} / \mathrm{ml})\end{array}$ & OD & $\%$ Hemolysis & $\%$ Retardation \\
\hline 1 & 100 & $0.12 \pm 0.01$ & $28.43 \pm 3.26$ & $44.36 \pm 6.36$ \\
\hline 2 & 200 & $0.096 \pm .018$ & $23.2 \pm 4.38$ & $53.93 \pm 8.84$ \\
\hline 3 & 300 & $0.06 \pm .08$ & $19.2 \pm 3.46$ & $61.86 \pm 7.26$ \\
\hline 4 & 400 & $0.05 \pm .07$ & $17 \pm 3.72$ & $66.6 \pm 7.27$ \\
\hline 5 & 500 & $0.04 \pm .06$ & $15.4 \pm 3.54$ & $69.8 \pm 6.91$ \\
\hline$I_{50}=160.86$ & & & \\
\hline
\end{tabular}

Each value represents the mean \pm SEM; $n=3$; As compared to the control: $P<0.01$, As compared to toxin treated : $P<0.01$.

Table-3: Effect of Standard Ascorbic Acid on Phenyl Hydrazine Induced Hemolysis

\begin{tabular}{|c|c|c|c|c|}
\hline S.No & $\begin{array}{c}\text { Concentration } \\
(\mu \mathrm{g} / \mathrm{ml})\end{array}$ & OD & $\%$ Hemolysis & $\%$ Retardation \\
\hline 1 & 100 & $0.11 \pm 0.01$ & $49.96 \pm 4.53$ & $47.56 \pm 4.76$ \\
\hline 2 & 200 & $.076 \pm .020$ & $34.8 \pm 9.23$ & $63.46 \pm 9.64$ \\
\hline 3 & 300 & $.09 \pm .047$ & $40.89 \pm 21.47$ & $57.06 \pm 22.50$ \\
\hline 4 & 400 & $.08 \pm .01$ & $37.83 \pm 5.47$ & $58.66 \pm 6.36$ \\
\hline 5 & 500 & $.063 \pm .029$ & $28.76 \pm 13.45$ & $69.76 \pm 14.10$ \\
\hline $\mathrm{IC}_{50}=163.5$ & & & \\
\hline
\end{tabular}

Each value represents the mean \pm SEM; $n=3$; As compared to the control: $P<0.01$, As compared to toxin treated: $P<0.01$.

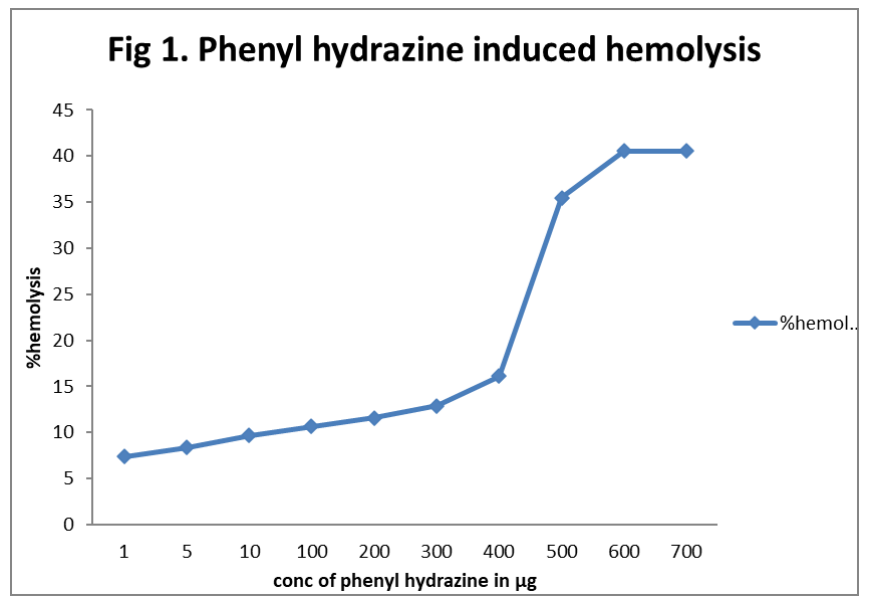

\section{Discussion}

Oxidative stress, an imbalance between free radical formation and the endogenous antioxidant protection system, primarily leads to buildup of oxidative damage,
Fig 2. Effect of rutin on phenyl hydrazine induced hemolysis

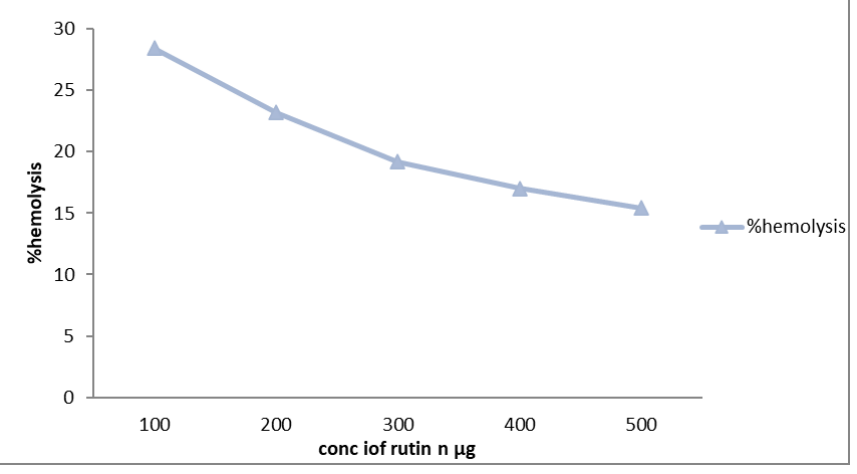

Fig 3. Effect of rutin on phenyl hydrazine induced hemolysis

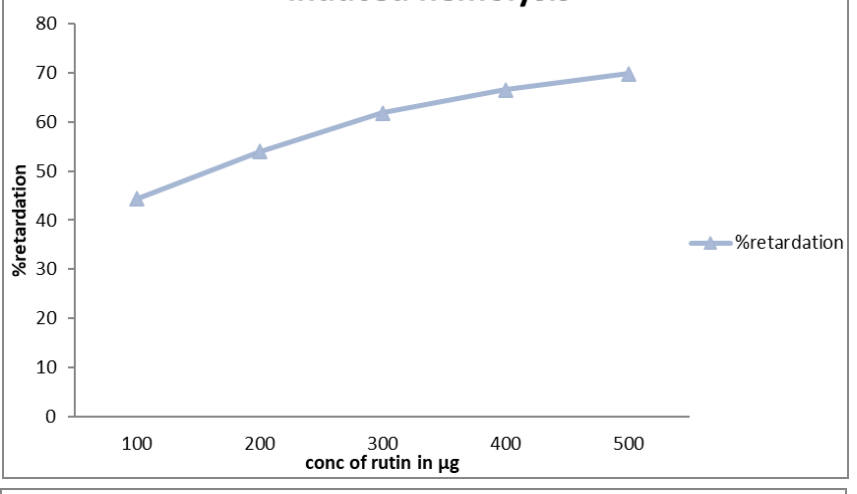

Fig 4. Effect of rutin on phenyl hydrazine induced hemolysis

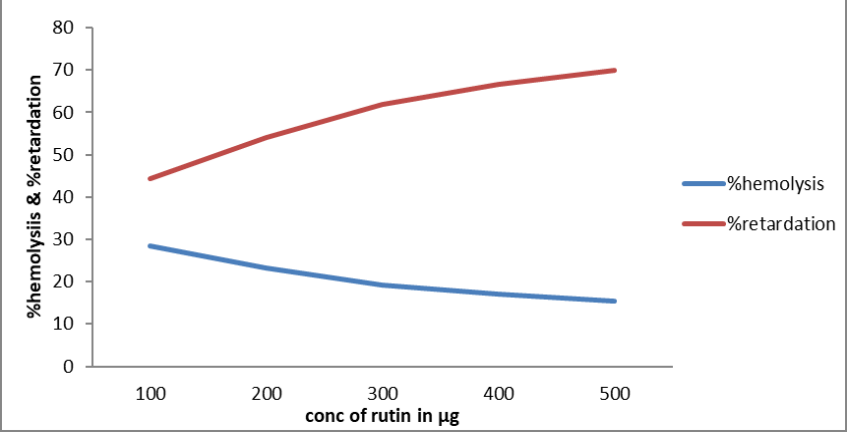

Fig 5. Ascorbic acid standard

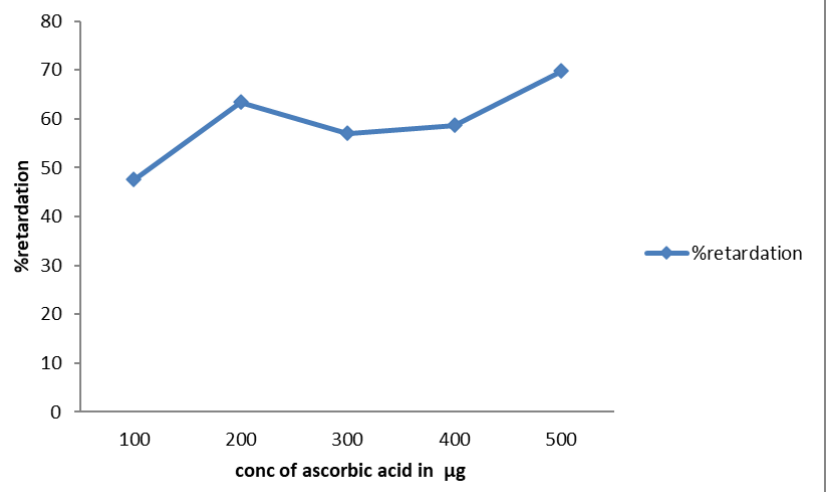

activation of stress-sensitive signaling pathways and generation of pathologic conditions such as 
cardiovascular disease, insulin resistance and metabolic syndrome [Abbas yavari et al., 2015]. Oxidative stress has been implicated in neurological diseases including Alzheimer's disease, Parkinson's disease, multiple sclerosis, memory loss, depression, production of vasoconstrictor molecules and primary salt retention by the kidney.

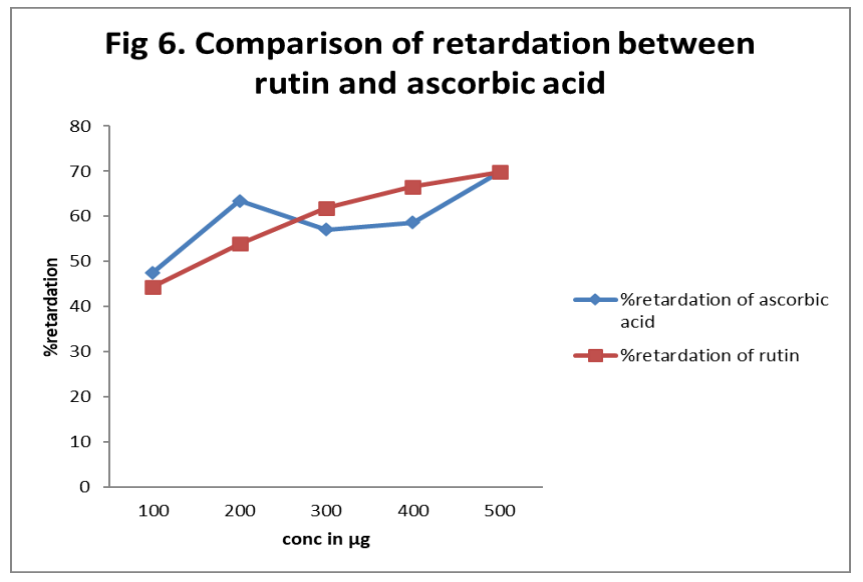

Oxidants play a significant role in the pathogenesis of a number of disorders such as inflammation, rheumatoid arthritis, asthma, psoriasis and contact dermatitis leading to oxidative stress, Free radicals can damage DNA and cause mutagenicity and cytotoxicity and thus play a key role carcinogenesis [Ashok shinde et al., 2012]. Erythrocytes are vulnerable to oxidative stress due to their high content of polyunsaturated lipids and transition metals, especially iron, which is known to catalyze free radicals generation via Fenton reaction [Chonthida Thephinlap et al., 2013]. These nontoxic antioxidant complexes of transition metals with Flavonoids could be of importance for the suppression of toxic effects of iron and copper ions released from ironor copper-containing proteins under certain pathological conditions [Igor Afanas et al., 2015].

\section{Conclusion}

The test compound Rutin has powerful retardation effect on phenyl hydrazine induced hemolysis. It is a phytochemical which acts as a potent anti-oxidant. RBC has the simplest structure which can be used as a very good model to identify the straight effect of a toxin on the cell membrane as well as protective effects by antidotes. Deterioration of the cell membrane in RBC can lead to cell lysis and release of haemoglobin in the medium. Destabilization of RBC membrane can be induced by autooxidation of Phenyl hydrazine which produces free radicals. The amount of hemolysis reveal the extent of toxicity. The results of the present investigation indicate that the possibility of employing the Rutin extract as an antioxidant substance to ameliorate the oxidative damage of cells. However, further attempts shall be made to investigate the possible protective effect of this extract against phenyl hydrazine induced cytotoxicity in vivo condition.

\section{References}

1. Taibur Rahman, Ismail Hosen, M.M Towhidul Islam, Hossain Uddin Shekhar "Oxidative stress and Human Health”, 2012, 997-1017.

2. Salim, Samina. "Oxidative stress and psychological disorders." Currentneuropharmacology 12 , 2, 2014, 140-147.

3. Abbas Yavari, Maryam Javadi, Parvin Mirmiran, and Zahra Bahadoran. "Exercise-induced oxidative stress and dietary antioxidants." Asian journal of sports medicine 6, 1, 2015.

4. Ashok Shinde, Jayashree Ganu, and Pankaja Naik. "Effect of free radicals \& antioxidants on oxidative stress: a review." Journal of Dental and Allied Sciences 1, $2,2012,63$.

5. Chonthida Thephinlap, Kanjana Pangjit, Maitree Suttajit, and Somdet Srichairatanakool. "Anti-oxidant properties and anti-hemolytic activity of Psidium guajava, Pandanous odorus and Rhinacanthus nasutus." Journal of Medicinal Plants Research 7, 25, 2013, 1849-1857.

6. Igor Afanas. "Mechanisms of superoxide signaling in epigenetic processes: relation to aging and cancer." Aging and disease 6, 3, 2015, 216. 